\title{
Neumonía complicada en pediatría, su manejo: un reto
}

\author{
Dra. Bertha Agudelo' \\ 1 Pediatra Neumóloga, Docente Universidad Tecnológica de Pereira. Colombia. \\ Presidente Asociación Colombiana de Neumología Pediátrica.
}

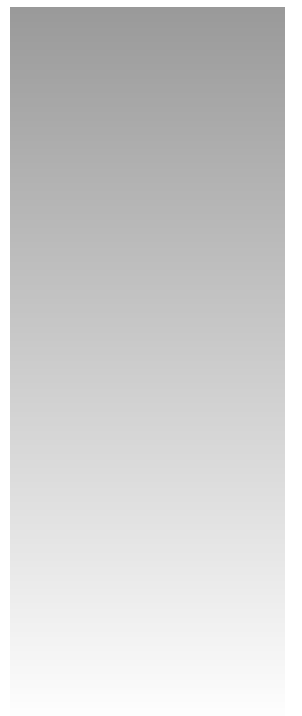

\section{TREATMENT OF EMPYEMA THORACIS AND NECROTIZING PNEUMONIA IN CHILDREN}

Complicated pneumonia with pleural effusion or empyema is a condition that its incidence has been increasing in recent years. Its early diagnosis and timely management reduce costs and shorten hospital stays for patients. The optimized diagnostic aids treatments. In recent years the intervention and fibrinolytic substances as VATS surgery have opened a positive outlook in handling controversies exist yet at the time of application. This article reviews these aspects.

Key words: Thoracis empiema, necrotizing pneumonia, children, management.

\section{RESUMEN}

La neumonía complicada con derrame pleural o empiema es una patología que ha ido incrementando su incidencia en los últimos años. Su diagnóstico temprano y su manejo oportuno disminuyen los costos y acortan las estancias hospitalarias de los pacientes. Las ayudas diagnósticas optimizan los tratamientos. En los últimos años la intervención con sustancias fibrinolíticas y las intervenciones quirúrgicas como la videotoracoscopia han abierto un panorama positivo en el manejo sin embargo, existen controversias en el momento de su aplicación. Este artículo hace una revisión de estos aspectos.

Palabras clave: Neumonía, empiema, videotoracoscopia, niños.

\section{INTRODUCCIÓN}

Las complicaciones de las neumonías conforman un grupo muy importante dentro de los niños ingresados a nuestros hospitales con patología torácica y suelen presentarse como lesiones broncopulmonares y pleurales complejas. Cuando la infección neumónica no se limita al parénquima pulmonar sino que se extiende a las pleuras y se presenta un empiema, se modifica radicalmente el curso clínico y el comportamiento de la entidad, constituyéndose la infección pleuropulmonar en un reto para su adecuado manejo, ya que no hay unificación en los criterios de clasificación lo que puede llevar a una confusión retraso en la toma de decisiones y a oportuna y adecuada intervención.

Las mayores complicaciones de la neumonía incluyen: neumotórax, (fístula broncopleural), neumonía necrosante y empiema. Las ultimas dos parece que han ido incrementando. La neumonía necrosante (NN) se puede sospechar por radiología, pero usualmente se diagnóstica por tomografía

Correspondencia: Bertha Inés Agudelo V. Docente Universidad Tecnológica de Pereira. La Julita Pereira. Colombia.

Tel 5763213443.

E-mail: berthagudelo@gmail.com

ISSN 07|8-332I Derechos reservados. computada. Ha sido un diagnóstico raro y, por lo tanto, es difícil calcular su real incidencia pero grandes series de casos sugieren que ha ido aumentando su frecuencia. Su diagnóstico se ha hecho más fácil y frecuente con la disponibilidad de estas técnicas imagenológicas. La neumonía necrosante usualmente coexiste con el empiema, el cual también ha ido incrementando su incidencia en diferentes partes del mundo(I). Los abscesos pulmonares aunque son complicaciones raras de la neumonía adquirida en la comunidad en niños, parece que igualmente han ido aumentando su frecuencia de presentación ${ }^{(2)}$.

\section{INCIDENCIA}

Las efusiones paraneumónicas se presentan en 1\% de los pacientes con neumonía adquirida en la comunidad, pero en los pacientes que ameritan hospitalizaciones se puede encontrar hasta en $40 \%$ de los casos. Se ha encontrado que el empiema ha aumentado su incidencia en los últimos años ${ }^{(3,4)}$.

En los Estados Unidos la incidencia de neumonía es de 30-40 por 100.000. En niños menores de 2 años la incidencia de empiema se duplicó en la última década desde 3,5 por 100.000 en 1996-1998 a 7 por 100.000 en el período 2005 al 2007(5). Similarmente en pacientes de 2 a 4 años la frecuencia se triplicó de 3,7 por 100.000 a 10,3 por 100.000 
durante el mismo período. La mortalidad en los niños es mucho menor que en los adultos y en estos últimos constituye un cuadro de difícil manejo y morbilidad.

\section{FACTORES PREDISPONENTES}

Hay algunos datos que sugieren que algunos niños tienen algunos factores predisponentes para estas formas severas de infección pulmonar. Estos factores incluyen; quistes congénitos, secuestros, bronquiectasias, desordenes neurológicos e inmunodeficiencia ${ }^{(6)}$. También existen datos de que ciertos serotipos de neumococos llevan con más frecuencia a la neumonía necrosante y a la formación de abscesos ${ }^{(7)}$. Igualmente que el Staphylococcus aureus productor de toxina Pantone Valentine leucocidine puede llevar a necrosis pulmonar severa con un alto riesgo de mortalidad ${ }^{(8)}$.

\section{ETIOLOGÍA}

La mayoría de los estudios epidemiológicos indican que el Streptococcus pneumoniae es el germen más frecuente como causal de neumonía complicada, antes y después de la introducción de la vacuna heptavalente antineumococo. Sin embargo, se ha notado un incremento de los casos por Staphylococcus aureus, algunos de ellos meticilino resistente, por lo cual se considera la segunda causa de neumonía complicada, seguido por otras especies de Streptococcus (S. pyogenes, S. millery), anaerobios, Haemophilus influenzae tipo b, Pseudomona aeruginosa, Mycoplasma pneumonia, y Mycobacterium tuberculosis (países con alta incidencia). También los virus como el adenovirus y la influenza ${ }^{(9,10)}$.

\section{FISIOPATOLOGÍA}

La clasificación del derrame pleural en efusión paraneumónica simple o empiema es de ayuda para entender la fisiopatología de la enfermedad pero no hay una evidencia clara de estrategias de manejo específicas en los diferentes estadios. El empiema es un derrame pleural purulento y constituye una fase en la progresión de un exudado inflamatorio. Inicialmente hay solamente inflamación a nivel de las pleuras, luego se inicia la acumulación de líquido que inicialmente es transparente y en la medida que aumenta el contenido de células se torna purulento. Se considera que el empiema tiene 3 ó 4 estadios en su evolución:

I. Estadio precolección que ocurra cuando la neumonía se asocia a inflamación de la pleura.

2. Estadio exudativo o efusión paraneumónica simple caracterizado por un líquido claro, con baja cantidad de células. Puede progresar o no a los siguientes estadios.

3. Estadio fibrinopurulento o efusión paraneumónica complicada, hay depósito de fibrina y de material purulento en el espacio pleural, incremento en la cantidad de células. Aparecen septos de fibrina.

4. Estadio de organización: engrosamiento de la pleura, la cual puede causar atrapamiento del pulmón y terminar en una enfermedad con patrón restrictivo crónico. Este estadio es raro en la época actual, especialmente en los niños pero se presenta cuando no se ha intervenido en forma temprana ${ }^{(1 \mid-13)}$.

El empiema se caracteriza por la colección de pus desde pocos a muchos centímetros cúbicos. Puede experimentar resolución espontánea, pero este desenlace no es el más frecuente, siendo así la organización con adherencias y tabiques que a menudo obliteran todo el espacio pleural. Durante la evolución de la enfermedad, se eleva la deshidrogenasa láctica, desciende el pH y la glucosa en el líquido pleural. Los criterios de Light para efusión pleural complicada incluyen: $\mathrm{pH}$ menor de 7,2, deshidrogenasa láctica mayor a $1.000 \mathrm{U}$, glucosa en el líquido menor de 40 mg/dl o menor del 25\% de la glicemia, tinción de Gram o cultivo del líquido positivos y la presencia de loculaciones o septos en las imágenes diagnósticas $^{(13)}$.

En 1995 Light propuso una clasificación nueva de los derrames pleurales donde correlaciona los hallazgos bioquímicos del líquido pleural con los hallazgos radiológicos y propuso 7 estadios diferentes desde el derrame no complicado hasta el empiema más difícil y una propuesta de intervenciones desde la observación hasta la decorticación ${ }^{(12)}$. En neumonía necrosante se ha encontrado el Streptococcus pneumoniae como el principal agente etiológico y de él los serotipos 14 y 3 son los más implicados. Se cree que se produce una gangrena pulmonar secundaria a una trombosis vascular(13).

\section{PRESENTACIÓN CLÍNICA}

Una fiebre que persiste a pesar del uso adecuado de antibiótico en un paciente con neumonía debe hacer sospechar al clínico la presencia de un empiema.

\section{HALLAZGOS RADIOLÓGICOS}

\section{Radiografía de tórax}

La radiografía de tórax es el examen que está más al alcance de todos los servicios de urgencias pero en ocasiones es muy difícil diferenciar el compromiso pulmonar de una colección. En estos casos podría ser de ayuda una radiografía lateral en decúbito para determinar si tiene una colección libre o loculada. En estos casos se necesitan otros exámenes de ayuda. Radiológicamente podemos encontrar estos tipos de lesiones en los pacientes con infección pleuropulmonar.

I. Derrame pleural (Figura I).

2. Lesiones hiperlúcidas hipertensas; neumotórax o lesiones intraparenquimatosas hipertensas (bulas).

3. Lesiones hiperlúcidas no hipertensas: abscesos, neumatoceles, neumonía abscedada o necrosante (Figura 2).

4. Lesiones secuelares: bronquiectasias, fibrotórax, bulas tabicadas, engrosamiento pleura.

\section{Ultrasonografía}

Es un examen fácil de realizar, en forma portátil, no es costoso y no genera irradiación. Se utiliza para localizar las efusiones y hacer drenajes. En series donde se compara el ultrasonido con la tomografía de tórax en el estudio del em- 


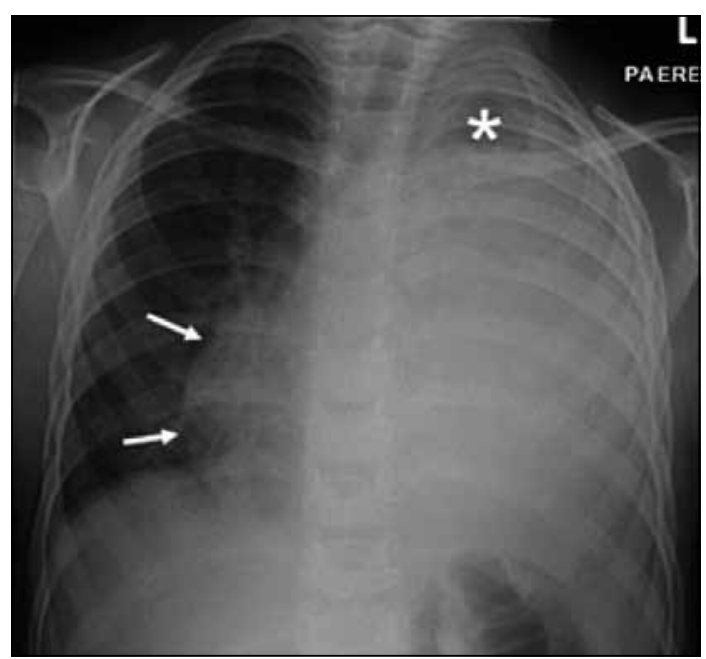

Figura I. Gran derrame pleural en un niño de 3 años. Efecto de masa con desviación del mediastino. piema no se encontró ventaja de la TAC sobre el ultrasonido al tomar decisiones ya que este último puede diferenciar con gran certeza las consolidaciones de la efusión, el volumen del líquido y la presencia de septos que son los requisitos indispensables a la hora de tomar decisiones. La desventaja, es un examen que depende del operador y en algunas instituciones pude ser problemático a la hora de tomar decisiones $^{(14)}$ (Figura 3).

\section{Tomografía de tórax}

No es más sensible que la ultrasonografía en demostrar septos, pero con contraste puede detectar mejor el engrosamiento pleural. La literatura reciente hace énfasis en que las dosis de radiación de la tomografía es acumulativa y puede aumentar el riesgo de cáncer. Se recomienda que se usen tomógrafos avanzados y con mínimas dosis de radiación, pero no se recomienda, por lo tanto, como examen de rutina en los pacientes con neumonía complicada ${ }^{(15)}$. La presencia de líquido en el espacio pleural se demuestra por la radiografía y la cantidad y calidad de líquido, igualmente los septos se detecta mejor por el ultrasonido. La tomografía sólo debería usarse en los casos difíciles.

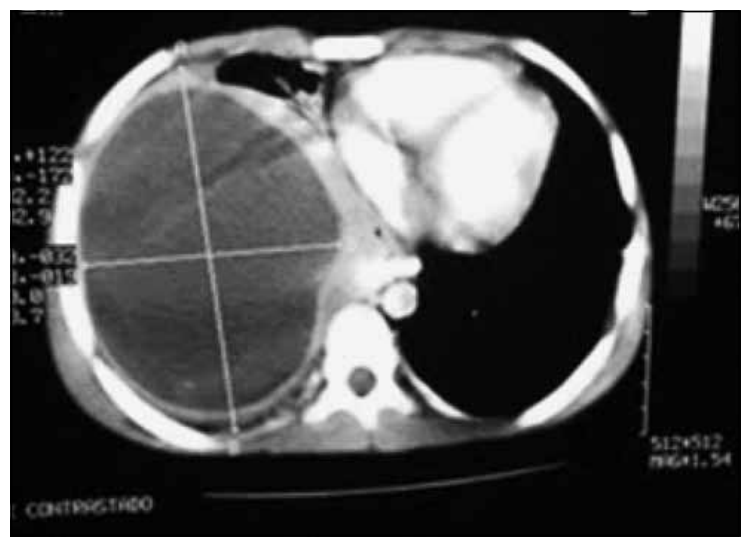

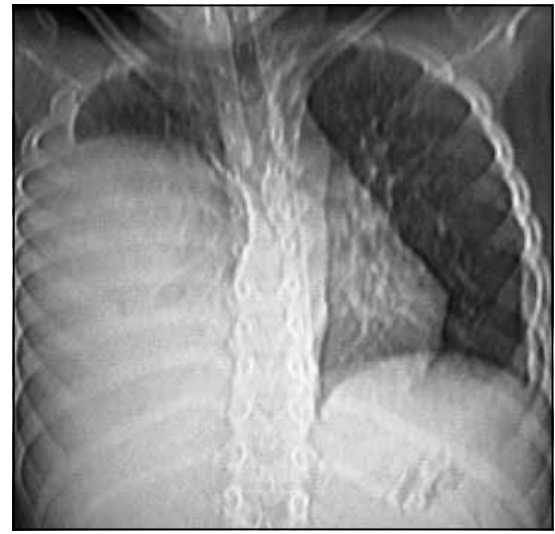

Figura 2. Absceso pulmonar en niño de 3 años de edad.

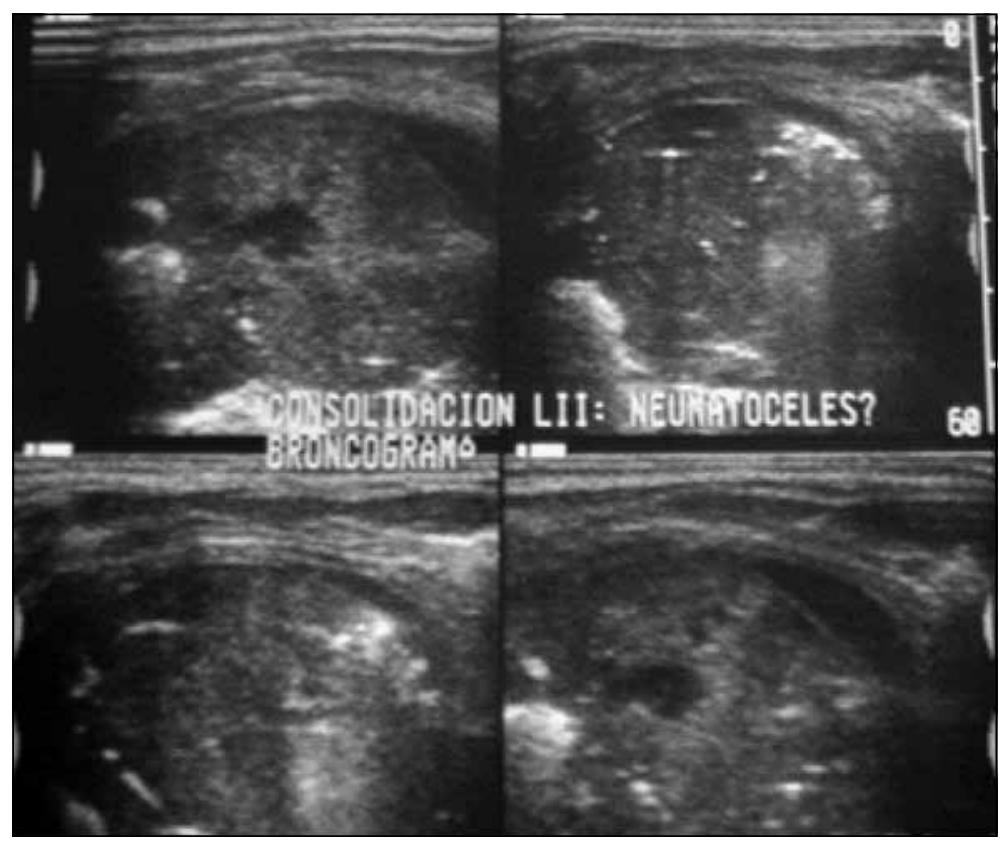

Figura 3. Ecografia de un niño de 3 años con neumonía complicada con derrame y con lesión necrosante intraparenquimatosa. 


\section{TRATAMIENTO DE LA NEUMONÍA COMPLICADA}

\section{Principios generales}

El manejo óptimo del empiema esta dirigido a cubrir los siguientes parámetros: Estado de la enfermedad, la bacteria aislada y de acuerdo a ellos, la terapia con antibióticos, la suplementación de oxígeno, el manejo de la fiebre y el dolor, el drenaje de la colección cuando es necesario, la hidratación y nutrición del paciente y el tiempo de remisión a un nivel más alto de atención.

\section{Manejo conservador}

El manejo inicial esta dirigido a la administración de antibióticos específicos, a dosis altas dirigidos contra los gérmenes detectados en hemocultivo o en el cultivo del líquido pleural, pero en la mayoría de los casos no se tiene un cultivo que lo detecte en forma rápida o estos son negativos por la administración previa de antibiótico. Se debe iniciar antibióticos de amplio espectro contra el neumococo y el $S$. aureus que son los gérmenes más frecuentes, sin tardanza. La mayoría de los empiemas no complicados causados por neumococo tienen buena respuesta la penicilina aunque sean detectado algunas cepas resistentes en los últimos tiempos.

En forma empírica se recomienda para hacer cobertura de los gérmenes más frecuentes: la oxacilina y una cefalosporina de tercera generación. Se ha reportado trabajos donde una cefalosporina de segunda generación como el cefuroxime tiene una buena cobertura para los dos gérmenes, y tiene la misma evolución y las mismas complicaciones que con los dos anteriores. Si se sospecha resistencia al antibiótico, una alternativa es cambiar a vancomocina o a clindamicina ${ }^{(16)}$.

En caso de sospecha de broncoaspiración se debe dar antibióticos contra anaerobios y S. milleri. Los macrólidos deben usarse cuando se sospeche que el agente causal es $M$. pneumoniae, pero no debe usarse rutinariamente.

En cuanto a la duración del tratamiento con antibióticos no existe evidencia exacta acerca de ello. En muchas instituciones se administra el antibiótico parenteral hasta que cese la fiebre o hasta que se retire el tubo de tórax. Se sigue con manejo de antibióticos orales de I a 4 semanas. Muchos casos de neumonía con efusión o empiemas pequeños resuelven solos con el antibiótico pero en muchos casos estos son muy grandes y comprometen el estado respiratorio del paciente y se hace necesario el drenaje con una toracentesis simple o con un tubo de tórax.

\section{¿Cuándo se debe hacer drenaje de la efusión paraneumónica?}

Se deben tener en cuenta 3 criterios:

I. Tamaño.

2. Síntomas.

3. Tabicaciones o presencia de septos.

I. El tamaño: En la radiografía de tórax tomada en decúbito es pequeño cuando tiene menos de $1 \mathrm{~cm}$ de colección intrapleural, moderado menos de $2 \mathrm{~cm}$ y grande mayor de $2 \mathrm{~cm}$. En la radiografía de tórax tomada de pie: pequeño cuando tiene opacificado menos de un cuarto del hemitórax, mediano cuando es más del cuarto pero menos de medio y grande cuando tiene opacificación de más de la mitad del hemitórax. La desviación del mediastino ya puede comprometer la función respiratoria y tener más síntomas. Según la literatura se considera en pediatría que un derrame pequeño hasta moderado podría ser manejado conservadoramente sin que se refleje mayor estancia hospitalaria o de presencia de complicaciones, pero que más grandes ya no serian manejados de acuerdo al tamaño sino a los síntomas y complicaciones.

2. Síntomas: Hace referencia a la fiebre, la taquipnea y la hipoxemia. El tamaño de la efusión tiene relación directa con la presencia de síntomas y estos últimos son los que tienen mayor relación con el pronóstico de estancia hospitalaria y las complicaciones.

3. Tabicaciones: Hace referencia a la presencia de material purulento y de fibrina, y normalmente en estas condiciones se hace necesario drenaje además del antibiótico.

Parámetros radiológicos y clínicos deben guiar la decisión de intervención en las efusiones paraneumónicas. La evacuación se debe hacer:

a. Derrames grandes.

b. Efusión con tabiques.

c. Efusión moderada con síntomas que no mejoran o que empeoran.

El drenaje debe realizarse mediante una simple toracentesis bajo sedación en el niño mayor. No se recomiendan toracentesis repetidas' ". Se debe usar tubos de nominación pequeña que igualmente son efectivos en efusiones loculadas o cuando se usa la fibronolisis. Idealmente la inserción del tubo de tórax debería hacerse en forma guiada bien sea por radiografía de tórax o idealmente mediante una ecografía. El tubo se debe retirar cuando el paciente esté afebril, que se haya controlado su cuadro de dificultad respiratoria, con buena re-expansión pulmonar y que el drenaje sea menor a $10 \mathrm{ml} / \mathrm{dí}^{(17)}$.

\section{Manejo conservador más terapia fibrinolítica}

Muchos de los derrames paraneumónicos se resuelven con el antibiótico y el drenaje con tubo de tórax, pero se presenta con alguna frecuencia que no sucede ello por la presencia de material fibrinoide y la presencias de loculaciones lo que complica el cuadro y se prolonga el manejo del paciente. En estos casos se ha recomendado el uso de sustancias fibrinolíticas, con el fin de cortar el fenómeno fisiopatológico y romper con los puentes de fibrina y remoción del material fibrinoide acumulado. Para tal fin se usan sustancias como la estreptoquinasa y la urokinasa, y el activador de plasminógeno tisular. Son pocos los estudios realizados en niños, pero se ha visto que al comparar el uso de estas sustancias vs la solución salina para el lavado de los empiemas si se reduce la estancia hospitalaria con estos últimos. Las dosis recomendadas son de $40.000 \mathrm{u}$ de urokinasa en $40 \mathrm{ml}$ de solución salina dos veces al día por 3 días, en niños mayores a $10 \mathrm{~kg}$ y para los niños menores 10.000 unidades en 10 cc de solución salina ${ }^{(18)}$. 


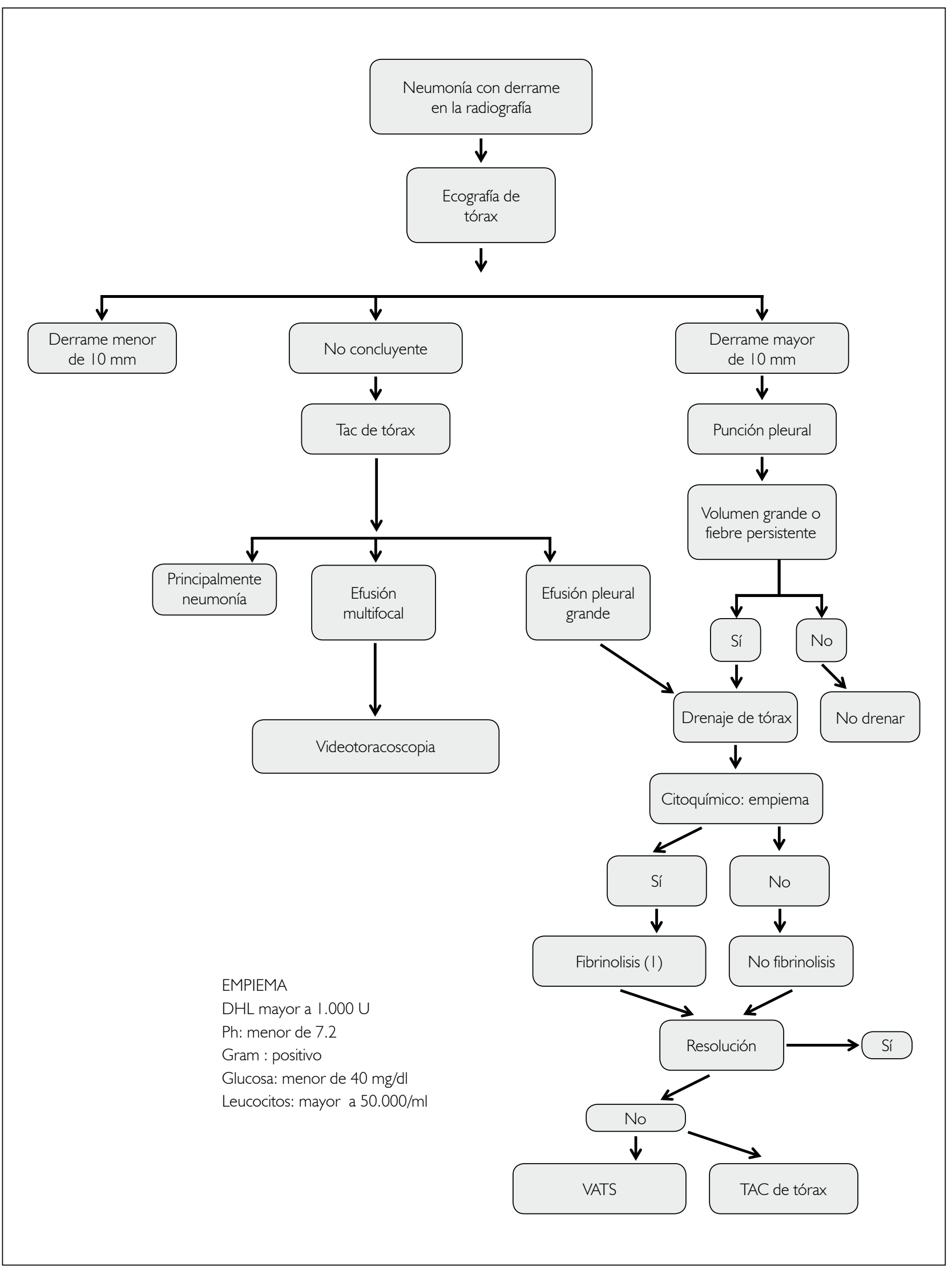

Figura 4. Algoritmo de manejo de la neumonía complicada con derrame pleural. 
El uso de las sustancias fibrinolíticas se ha reportado como un procedimiento seguro y bien tolerado pero se ven mayores complicaciones con el uso de la estreptoquinasa por causar mayores respuestas alérgicas, al ser extraído de lisados de bacterias al igual que hemorragias (hemotórax y hematuria) y dolor torácico. La urokinasa es mejor tolerada por ser extraída de cultivos de células humanas, con menores respuestas alérgicas y sistémicas ${ }^{(19,20)}$. Otra sustancia que se ha utilizado como fibrinolítico en los últimos años es la dornasa alfa (rDNAsa) que tiene efectos mucoliticos y se ha usado nebulizada y en instilación en tubo orotraqueal, pero su uso a nivel intrapleural apenas se ha ido desarrollando en unos

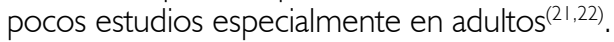

\section{Manejo quirúrgico}

El manejo del niño con empiema, tradicionalmente se ha hecho con manejo conservador inicial y cuando este falla, se lleva a un procedimiento quirúrgico. El objetivo del manejo es remover el material purulento, romper los septos de fibrina, controlar la infección, lograr la reexpansión y buen funcionamiento del pulmón.

\section{¿Cuándo se debe hacer?}

La decisión de la intervención se debe tomar cuando falla el tratamiento conservador, o sea que persista la fiebre y el esta séptico del paciente, cuando aumenten los requerimientos de oxígeno y en los casos de loculación que no mejoren con el tratamiento fibrinolítico. Los procedimientos utilizados son: minitoracotomía, toracotomía con decorticación y la videotoracoscopia asistida (VATS). La menos invasiva y que ha tenido resultados muy favorables en los últimos años es la VATS, con la cual se logra el mismo drenaje que con la minitoracotomía, siendo menos cruenta; la evolución del paciente cuando se hace en forma temprana es muy buena y se acorta en forma notoria la estancia hospitalaria. Al comparar la VATS con la terapia fibrinolítica los estudios que se han hecho han demostrado que tiene una evolución similar pero la decisión de cual escoger depende de la posibilidad de conseguir un cirujano entrenado en estas técnicas quirúrgicas ${ }^{(23)}$.

\section{¿Cuál es el manejo del absceso y de la neumonía necrosante?}

Cuando se documenta una lesión abscedada en el pulmón, se recomienda de entrada el tratamiento conservador con antibióticos solamente. Pero si tiene una enfermedad o anomalía de la vía aérea se recomienda la cirugía de ella tan pronto se haya controlado el proceso infeccioso. Si se documenta el absceso en una posición muy periférica y no tiene comunicación con la vía aérea, se podría pensar en un drenaje con catéter direccionado por una tomografía o por un ultrasonido. En algunos estudios retrospectivos se vio que la intervención acorta la estancia hospitalaria y facilita la recuperación del paciente ${ }^{(24)}$.

La necrosis pulmonar representa la peor forma de enfermedad parenquimatosa y no se puede tratar con debriadamiento pleural. No hay datos publicados que indiquen intervenir al paciente por lo cual en estos casos se recomienda el tratamiento conservador con antimicrobianos. La intervención en un tejido necrótico puede llevar a sangrados severos e incontrolables, fístulas broncopleurales y altos riesgos de neumotórax severos.

Determinar la extensión y severidad de la enfermedad parenquimatosa en relación a la enfermedad pleural es definitivo antes de tomar cualquier decisión terapéutica. Si la necrosis es difusa más que el derrame pleural se debe enfocar el paciente en el manejo de la infección en forma conservadora y luego determinar si se necesita un procedimiento adicional.

En resumen, los abscesos parenquimatosos y la necrosis pulmonar deben ser de manejo conservador. Si la fibrinolisis o la VATS es necesaria por enfermedad pleural concomitante se deben tener precauciones con la manipulación pulmonar.

\section{REFERENCIAS}

I. Weigl JA, Puppe W, Belke O, et al. Population-based incidence of severe pneumonia in children in Kiel, Germany. Klin Padiatr 2005; 217: $211-9$.

2. Sowicki GS, Lu FL, Valim C, et al. Necrotising pneumonia is an increasingly detected complication of pneumonia in children. Eur Respir J 2008; 31: |285-9|.

3. Chonmaitree T, Powell KR. Parapneumonic pleural effusion and empyema in children. Review of a 19-year experience, 1962e 1980. Clin Pediatr 1983; 22: 4I4-9.

4. Buckingham SC, King MD, Miller ML. Incidence and etiologies of complicated parapneumonic effusions in children, 1996 to 2001. Pediatr Infect Dis J 2003; 22: 499-504.

5. Grijalva CG, Nuorti JP, Zhu Y, et al. Increasing Incidence of Empyema Complicating Childhood Community Acquired Pneumonia In The United States. Clin Infect Dis 20 I0; 50: 805-I3.

6. Byington $C L$, Spencer $L Y$, Johnson TA, et al. An epidemiological investigation of a sustained high rate of pediatric parapneumonic empyema: risk factors and microbiological associations. Clin Infect Dis 2002; 34: 434-40.

7. Ramphul N, Eastham KM, Freeman R, et al. Cavitatory lung disease complicating empyema in children. Pediatr Pulmonol 2006; 41: 750-3.

8. Gillet $Y$, Vanhems $P$, Lina $G$, et al. Factors predicting mortality in necrotizing community acquired pneumonia caused by Staphylococcus aureus containing Panton-Valentine leukocidin. Clin Infect Dis 2007; 45: 315-21.

9. Buckingham SC, King MD, Miller ML. Incidence and etiologies of complicated parapneumonic effusions in children, 1996 to $200 \mathrm{I}$. Pediatr Infect Dis J 2003; 22: 499-504.

10. Proesmans M, De Boeck K. Clinical practice: treatment of childhood empy- ema. Eur J Pediatr 2009; 168: 639-45.

I I. Balfour-Lynn IM, Abrahamson E, Cohen G, et al. BTS guidelines for the management of pleural infection in children. Thorax 2005; 60 (Suppl I): il-i2I.

12. Light RW. A new classification of parapneumonic effusions and empyema. Chest 1995; 108: 299-301.

13. Hsieh YC, Hsiao Ch, Tsao P, et al. Necrotizing Pneumococcal Pneumonia in Children: The Role of Pulmonary Gangrene. Pediatr Pulmonol 2006: 41: 623-9.

14. Kurian J, Levin TL, Han BK, Taragin, Weinstein S. Comparison of Ultrasound and CT in the Evaluation of Pneumonia Complicated by Parapneumonic Effusion in Children. AJR 2009; 193: 1648-54. 
15. Bradley JS, Byington CL, Shah SS, et al. Executive Summary: The management of community acquired pneumonia in infants and children older than 3 months of age: Clinical practice guidelines by the Pediatric Infectious Disease Society and the Infectious Disease Society of America. Clin Infect Dis 201 I; 53: 617-30.

16. Agudelo B, Arango M, Cala L, Manotas M, Vásquez C, Agudelo $\mathrm{Bl}$, et al. Guía de práctica clínica en el tratamiento del niño con neumonía adquirida en la comunidad. Asociación Colombiana de Neumología Pediátrica Guías de práctica clínica 2010 Guía No. 5 http://issuu.com/acnp/docs/neumonia l?e=379079 I/260645 I .

17. Barnes NP, Hull J, Thomson AH. Medical management of parapneumonic pleural disease. Pediatr Pulmonol 2005; 39: I2734.

18. Barbato A, Panizzolo C, Monciotti C, et al. Use of urokinase in childhood pleural empyema. Pediatr Pulmonol 2003; 35: 50-5.

19. Stefanutti G, Ghirardo V, Barbato A, et al. Evaluation of a pediatric protocol of intrapleural urokinase for pleural empyema: a prospective study. Surgery 20 10; 148: 589-94.

20. Tokuda Y, Matsushima D, Stein GH, et al. Intrapleural fibrinolytic agents for empyema and complicated parapneumonic effusions: a meta-analysis. Chest 2006; 129: 783-90.

21. Simpson G, Roomes D, Reeves B. Successful treatment of empyema thoracis with human recombinant deoxyribonuclease. Thorax 2003; 58: 365-6.

22. Rahman NM, Maskell NA, West A, et al. Intrapleural use of tissue plasminogen activator and DNase in pleural infection. N Engl J Med 2011; 365: 518-26.

23. Paraskakis E, Vergadi E, Cathzimichael A, Bouros D. Current evidence for the management of paediatric parapneumonic effusions. Curr Med Res Opin 2012; 28: 1179 -92.

24. Patradoon-Ho P, Fitzgerald DA. Lung abscesses in children. Paediatr Respir Rev 2007; 8: 77-84. 